\title{
A UNIFIED APPROACH TO UNIFORM REAL APPROXIMATION BY POLYNOMIALS WITH LINEAR RESTRICTIONS
}

\author{
BY \\ BRUCE L. CHALMERS
}

\begin{abstract}
Problems concerning approximation of real-valued continuous functions of a real variable by polynomials of degree smaller than $n$ with various linear restrictions have been studied by several authors. This paper is an attempt to provide a unified approach to these problems. In particular, the notion of restricted derivatives approximation is seen to fit into the theory and includes as special cases the notions of monotone approximation and restricted range approximation. Also bounded coefficients approximation, $\varepsilon$-interpolator approximation, and polynomial approximation with interpolation fit into our scheme.
\end{abstract}

1. Introduction, preliminaries, and examples. Problems concerning approximation of real-valued continuous functions of a real variable by polynomials of degree smaller than $n$ with various linear restrictions have been studied by several authors. This paper is an attempt to provide a unified approach to these problems. In particular, the notion of restricted derivatives approximation is seen to fit into the theory and includes as special cases the notions of monotone approximation and restricted range approximation. Also bounded coefficients approximation, $\varepsilon$-interpolator approximation, and polynomial approximation with interpolation fit into our scheme. (See examples in this section.)

Let $E$ denote a compact subset of the real line and let $C E$ denote the real space of real-valued continuous functions on $E$ with the usual supremum norm $\|\cdot\|$. Let $V^{n}$ be an $n$-dimensional subspace of $C E$. For some fixed compact topological index space $A$, let $\left\{L^{\alpha}\right\}_{\alpha \in A}$ be a set of linear functionals defined on $V^{n}$, such that, for each $p$ in $V^{n}, L^{\alpha} p$ is a continuous function on $A$. Now set

$$
V_{0}^{n}=\left\{p \in V^{n} ; l_{\alpha} \leqq L^{\alpha} p \leqq u_{\alpha}, \alpha \in A\right\},
$$

where $l_{\alpha}$ and $u_{\alpha}$ are extended-real-valued functions on $A$ and $l_{\alpha}<+\infty, u_{\alpha}>-\infty$, $l_{\alpha}$ is upper semicontinuous, $u_{\alpha}$ is lower semicontinuous, and $l_{\alpha} \leqq u_{\alpha}$. We assume, moreover, that $l_{\alpha}=u_{\alpha}$ implies $\alpha$ is an isolated point of $A$.

Let $e_{x}$ represent point evaluation at $x$ in $E$. Now consider any fixed $f$ in $C E-V_{0}^{n}$ with the restriction that if $L^{\alpha}=e_{x}$ for some $\alpha$ in $A$ and some $x$ in $E$, then $l_{\alpha} \leqq f(x)$

Presented to the Society, September 3, 1971; received by the editors March 26, 1971 and, in revised form, August 10, 1971.

AMS 1970 subject classifications. Primary 41A50; Secondary 41A10.

Key words and phrases. Approximation with linear restrictions, polynomials of best approximation, extremal sets, uniqueness of best approximation, generalized Haar condition.

Copyright (C) 1972, American Mathematical Society 
$\leqq u_{\alpha}$. We will be concerned with approximating $f$ by elements of $V_{0}^{n}$ but first we will make a final Haar-like assumption on $V^{n}$, which we develop as follows. To each $L^{\alpha}, \alpha \in A$, we associate a set (possibly empty) of elements $\left\{M^{\beta}\right\}_{\beta \in B_{\alpha}}$ in the dual of $V^{n}$ such that, if $p$ is in $V_{0}^{n}$, then $L^{\alpha} p=l_{\alpha}$ (or $u_{\alpha}$ ) implies that, for each $\beta$ in the index set $B_{\alpha}, M^{\beta} p=m_{\beta}$ (or $n_{\beta}$ ), where $m_{\beta}$ (or $n_{\beta}$ ) is some real number depending only on $\beta$.

Definition. For $p$ in $V_{0}^{n}$ a set $\mathscr{S}=\left\{L^{\alpha}\right\}_{\alpha \in I_{1}} \cup\left\{M^{\beta}\right\}_{\beta \in I_{2}} \cup\left\{e_{x}\right\}_{x \in I_{3}}$ of $t$ ( $t$ may be infinite) symbols representing distinct elements in the dual of $V^{n}$ (where $I_{1} \subset A$, $I_{3} \subset E$, and $I_{2} \subset \bigcup_{\alpha \in I_{1}} B_{\alpha}$ ) is called an extremal set for $f$ and $p$ of order $t$ if

(i) $L^{\alpha} p=u_{\alpha}\left(\right.$ or $\left.l_{\alpha}\right), \alpha \in I_{1}$,

(ii) $|(f-p)(x)|=\|f-p\|, x \in I_{3}$,

(iii) $e_{x} \notin\left\{L^{\alpha}\right\}_{\alpha \in I_{1}}$ if $|(f-p)(x)|=\|f-p\|$,

(iv) $L^{\alpha} \notin\left\{M^{\beta}\right\}_{\beta \in I_{2}}$ if $L^{\alpha} p=u_{\alpha}$ (or $l_{\alpha}$ ).

Definition. If, for some $p$ in $V_{0}^{n},\|f-p\|$ equals the infimum of $\|f-h\|$ where $h$ runs through $V_{0}^{n}$, then $p$ is called a best approximation in $V_{0}^{n}$ to $f$.

Definition. $V^{n}$ is generalized Haar with respect to $f$ and $p$, where $p$ is a best approximation in $V_{0}^{n}$ to $f$, provided that the maximal extremal set for $f$ and $p$ of order $t$ contains $t^{\prime}=\min (t, n)$ elements which are linearly independent in the dual of $V^{n}$.

The maximal extremal set for $f$ and $p$ is in fact obtained by taking

$$
\begin{aligned}
& I_{3}=\{x \in E ;|(f-p)(x)|=\|f-p\|\} \\
& I_{1}=\left\{\alpha \in A ; L^{\alpha}=l_{\alpha}\left(\text { or } u_{\alpha}\right)\right\}-\left\{\alpha ; L^{\alpha}=e_{x} \text { for some } x \in I_{3}\right\}
\end{aligned}
$$

and

$$
I_{2}=\bigcup_{\alpha \in I_{1}} B_{\alpha}-\left\{\beta ; M^{\beta}=L_{\alpha} \text { for some } \alpha \in I_{1}\right\} .
$$

Definition. $V^{n}$ is generalized Haar if $V^{n}$ is generalized Haar with respect to $f$ and $p$ for all admissible pairs $f$ and $p$.

If $A$ is empty then any extremal set consists of just point evaluations and the generalized Haar condition on $V^{n}$ reduces to the ordinary Haar condition on $V^{n}$, i.e. any $n$ point evaluations are linearly independent in the dual of $V^{n}$.

We will show in particular that, if $V_{0}^{n}$ is not empty and $V^{n}$ is generalized Haar with respect to $f$ and $p$, then $p$ is the unique best approximation in $V_{0}^{n}$ to $f$.

We now give several examples, the first two of which will be seen to be special cases of Example 3, which we will show fits into the general scheme given above. In the sequel $P_{n-1}$ will represent the space of real polynomials of degree no larger than $n-1$, i.e. $P_{n-1}=\left\{c_{0}+c_{1} x+\cdots+c_{n-1} x^{n-1}\right\}$.

ExAmple 1 (Monotone APPROXimation). Let $\left(\varepsilon_{0}, \varepsilon_{1}, \ldots, \varepsilon_{q}\right)$ be a $(q+1)$-tuple with elements equal to \pm 1 and let $\left\{k_{i}\right\}_{i=0}^{q}$ be a fixed set of $q+1$ integers satisfying $0<k_{0}<k_{1}<\cdots<k_{q} \leqq n-1$. "Monotone approximation" in $C[a, b]$ by the set $N=\left\{p \in P_{n-1} ; \varepsilon_{i} p^{\left(k_{i}\right)}(x) \geqq 0\right.$ on $\left.[a, b], i=0,1, \ldots, q\right\}$ has been studied by $O$. Shisha [13], G. G. Lorentz and K. L. Zeller [6], R. A. Lorentz [7], and J. A. Roulier 
[10]. Uniqueness of best approximation is shown in [6] when $q=0$ and in [7] for arbitrary $q$.

Example 2 (Restricted Range Approximation). Approximation in $C[a, b]$ by the set $N=\left\{p \in P_{n-1} ; l(x) \leqq p(x) \leqq u(x), x \in[a, b]\right\}$, where $l(x)$ and $u(x)$ are extended-real-valued functions, upper and lower semicontinuous respectively, and $l(x) \leqq f(x) \leqq u(x)$ on $[a, b]$, has been investigated by G. D. Taylor and others. With the assumption that $l(x)<u(x)$ on $[a, b]$, uniqueness of best approximation is shown in [14] for $l(x), u(x)$ continuous on $[a, b]$.

These two examples are seen to be special cases of the following example.

Example 3 (Restricted Derivatives Approximation). Let $\left\{k_{i}\right\}_{i=0}^{q}$ be a fixed set of $q+1$ integers satisfying $0 \leqq k_{0}<k_{1}<\cdots<k_{q} \leqq n-1$. Consider approximation in $C[a, b]$ by the set $N=\left\{p \in P_{n-1} ; l_{i}(x) \leqq p^{\left(k_{i}\right)}(x) \leqq u_{i}(x), i=0,1, \ldots, q\right\}$, where $l_{i}(x)<+\infty$ and $u_{i}(x)>-\infty$ are extended-real-valued functions, upper and lower semicontinuous respectively, and $l_{i}(x)<u_{i}(x)$ on $[a, b], i=0,1, \ldots, q$. Also assume that if $k_{0}=0$ then $l_{0}(x) \leqq f(x) \leqq u_{0}(x)$, and if $k_{i}>0$, then $l_{i}(x)$ and $u_{i}(x)$ are either differentiable or identically infinite on $[a, b]$. As an application of our theory, we will obtain uniqueness of best approximation for restricted derivatives approximation in case $N \neq \varnothing$.

Restricted derivatives approximation fits into our scheme as follows. First let $E=[a, b]$ and let $A=\bigcup_{i=0}^{q}\{(x, i) ; x \in E\}$ endowed with the obvious topology. Let $V_{n}=P_{n-1}$. Then for each $\alpha=(x, i) \in A$, we have $L^{\alpha} p=p^{\left(k_{i}\right)}(x)$ and to each such $L^{\alpha}$, where $k_{i}>0$ and $a<x<b$, we associate the set of one element $\left\{M^{\alpha}\right\}$ where $M^{\alpha} p=p^{\left(k_{i}+1\right)}(x)$. Next we set $l_{\alpha}=l_{i}(x), u_{\alpha}=u_{i}(x)$ for all $\alpha=(x, i)$ in $A$, and we find that $N=V_{0}^{n}=\left\{p \in V^{n} ; l_{\alpha} \leqq L^{\alpha} p \leqq u_{\alpha}, \alpha \in A\right\}$. It is clear that if $p$ is in $V_{0}^{n}$, then $L^{\alpha} p=l_{\alpha}\left(\right.$ or $\left.u_{\alpha}\right)$ implies $M^{\alpha} p=p^{\left(k_{i}+1\right)}(x)=l_{i}^{(1)}(x)\left(\right.$ or $\left.u_{i}^{(1)}(x)\right)=m_{\alpha}\left(\right.$ or $\left.n_{\alpha}\right)$ if $k_{i}>0$ and $a<x<b$.

One can check that the remaining conditions hold. Surely $L^{\alpha} p=p^{\left(k_{i}\right)}(x)$ is a continuous function on $A$ for each $p$ in $V^{n}$, and if $L^{\alpha}=e_{x}$ for some $\alpha$ in $A$ and some $x$ in $E$, then $l_{\alpha} \leqq f(x) \leqq u_{\alpha}$. The fact that $V^{n}$ is generalized Haar will be demonstrated in $\S 3$.

EXAMPLE 4. Let $E$ be any compact subset of the real line and let $V^{n} \subset C E$. Further consider $s$ elements $L^{1}, L^{2}, \ldots, L^{s}$ in the dual of $V^{n}$ such that $\left\{L^{1}, L^{2}, \ldots, L^{r}, e_{x_{r+1}}, e_{x_{r+2}}, \ldots, e_{x_{n}}\right\}$ is an independent set for every choice of $n-r$ points $\left\{x_{r+1}, x_{r+2}, \ldots, x_{n}\right\}, r \leqq s$, provided $e_{x_{j}} \neq L^{i}, i=1,2, \ldots, r ; j=r+1$, $\ldots, n$. Let $V_{0}^{n}=\left\{p \in V^{n} ; l_{i} \leqq L^{i} p \leqq u_{i}, i=1,2, \ldots, s\right\}$. (In the case $l_{i}=u_{i}$ and $L^{i} \neq e_{x}$ for any $x \in E, i=1,2, \ldots, s$, uniqueness of best approximation by elements in $V_{0}^{n}$ was shown by Rivlin and Shapiro [9, p. 679].)

This example fits into our scheme where $A=\{1,2, \ldots, s\}$ with the discrete topology. Then we take $M^{\alpha}=\varnothing$ for all $\alpha$ in $A$, and the rest of the conditions follow immediately. For example, since any set of distinct elements $\left\{L^{1}, \ldots, L^{r}, e_{x_{r+1}}, \ldots\right.$, $\left.e_{x_{n}}\right\}$ is linearly independent, $r \leqq s$, it follows trivially that $V^{n}$ is generalized Haar.

Note. In the case $l_{i}=u_{i}, i=1,2, \ldots, s$, a maximal extremal set $\mathscr{S}$ must contain 
all $L^{i}, i=1,2, \ldots, s$, and so the condition on $V^{n}$ can be relaxed to requiring only that $\left\{L^{1}, L^{2}, \ldots, L^{s}, e_{x_{s+1}}, \ldots, e_{x_{n}}\right\}$ is independent (as in [9]).

Applying Example 4, we have the following three specific examples:

EXAmple 5 (Bounded Coefficients APPROXimation). Let $\left\{k_{i}\right\}_{i=0}^{q}$ be a fixed set of $q+1$ integers satisfying $0 \leqq k_{0}<k_{1}<\cdots<k_{q} \leqq n-1$. Approximation in $C[0,1]$ by the set $N=\left\{p \in P_{n-1} ; l_{i} \leqq p^{\left(k_{i}\right)}(0) \leqq u_{i}, i=0,1, \ldots, q\right\}$ has been studied by J. A. Roulier and G. D. Taylor [11] in which they proved uniqueness of best approximation provided, in the case $k_{0}=0$, that $l_{0} \leqq f(0) \leqq u_{0}$. (Letting $L^{i} p$ $=p^{\left(k_{i}\right)}(0), i=0,1, \ldots, q$, we have the linear independence required in Example 4 as a result of Theorem $\mathrm{C}$ in \$3.)

EXAMPle 6 ( $\varepsilon$-INTERPOlator APProximation). Fix $x_{1}<x_{2}<\cdots<x_{k}$ in $E$ with $k \leqq n$ and let $\varepsilon>0$ be given. Let $V^{n}$ be an $n$-dimensional Haar subspace of $C E$. Then $p$ in $V^{n}$ is said to $\varepsilon$-interpolate $f$ at $x_{1}, x_{2}, \ldots, x_{k}$ provided $\left|f\left(x_{i}\right)-p\left(x_{i}\right)\right| \leqq \varepsilon$ for each $x_{i}$. G. D. Taylor [15] proved uniqueness of best approximation of $f$ by $\varepsilon$-interpolating elements in $V^{n}$.

Example 7 (Polynomial Approximation with Interpolation). Fix $x_{1}<x_{2}$ $<\cdots<x_{k}$ in $[a, b]$ with $k \leqq n$. Let $V^{n}=P_{n-1}$. Then let $K=\left\{p \in P_{n-1} ; p^{(j)}\left(x_{i}\right)=a_{i j}\right.$, $1 \leqq i \leqq k$ and $\left.0 \leqq j \leqq m_{i}-1\right\}$ where $\left\{a_{i j}\right\}$ is a set of real numbers and $\left\{m_{i}\right\}_{i=1}^{k}$ is a set of positive integers such that $\sum_{i=1}^{k} m_{i}<n$. Uniqueness of best approximation to $f \in\left\{f \in C[a, b] ; f\left(x_{i}\right)=a_{i 0}, 1 \leqq i \leqq k\right\}$ by elements of $K$ was proved in [5]. (Letting $L^{i j} p=p^{(j)}\left(x_{i}\right)$, we have the linear independence required in Example 4 as a result of Theorem $C$ in $\$ 3$ and the note following Example 4.) Also from Theorem D in $\S 3$ it is clear how we can generalize the set $K$ of Example 7.

\section{Main results.}

THEOREM 1. If $V_{0}^{n}$ is not empty, then there exists a best approximation in $V_{0}^{n}$ to $f$.

Proof. Let $\rho$ be the infimum of the values $\|f-h\|, h$ in $V_{0}^{n}$. Pick $h_{0}$ in $V_{0}^{n}$. Then $\|f-h\|>\left\|f-h_{0}\right\| \geqq \rho$ for all $h$ such that $\left\|h-h_{0}\right\|>2\left\|f-h_{0}\right\|$. Thus the continuous function $\|f-h\|$ achieves its infimum on $\left\{h \in V_{0}^{n} ;\|h\| \leqq 2\left\|f-h_{0}\right\|+\left\|h_{0}\right\|\right\}$, since this set is a closed and bounded subset of the finite-dimensional space $V^{n}$ and hence compact.

THEOREM 2. If $V^{n}$ is generalized Haar with respect to $f$ and $p$, then there exists an extremal set for $f$ and $p$ of order $n+1$.

Proof. Let $\|f-p\|=\rho$. Suppose there does not exist an extremal set of order $n+1$. Let $\mathscr{S}=\left\{L^{\alpha_{v}}\right\}_{v=1}^{r} \cup\left\{M^{\beta_{\mu}}\right\}_{\mu=r+1}^{s} \cup\left\{e_{x_{i}}\right\}_{i=s+1}^{t}$ be of maximal order $t \leqq n$.

Recall that $l_{\alpha}<+\infty, u_{\alpha}>-\infty, l_{\alpha}$ is upper semicontinuous, $u_{\alpha}$ is lower semicontinuous, and $l_{\alpha}<u_{\alpha}$ except possibly at isolated points of $A$. Hence it is easily seen that we can find a continuous function $c_{\alpha}$ such that either $l_{\alpha}<c_{\alpha}<u_{\alpha}$ or $l_{\alpha}=c_{\alpha}=u_{\alpha}$ on $A$. (This is the "Einschiebungssatz" of Hausdorff.) 
Further define the function $\left\{r_{\alpha}\right\}_{\alpha \in A}$ by

$$
\begin{aligned}
r_{\alpha} & =\min \left(u_{\alpha}-c_{\alpha}, c_{\alpha}-l_{\alpha}\right) & & \text { if }-\infty<l_{\alpha} \leqq u_{\alpha}<+\infty, \\
& =c_{\alpha}-l_{\alpha} & & \text { if } u_{\alpha}=+\infty, l_{\alpha}>-\infty, \\
& =u_{\alpha}-c_{\alpha} & & \text { if } l_{\alpha}=-\infty, u_{\alpha}<+\infty, \\
& =\infty & & \text { if } l_{\alpha}=-\infty, u_{\alpha}=+\infty .
\end{aligned}
$$

Next, because of the independence of the functionals in $\mathscr{S}$, there exists a nonzero element $q$ of $V^{n}$ such that $L^{\alpha_{v}} q=-\operatorname{sgn}\left(L^{\alpha_{v}} p-c_{\alpha_{v}}\right), \nu=1, \ldots, r$, and $q\left(x_{i}\right)$ $=\operatorname{sgn}\left((f-p)\left(x_{i}\right)\right), i=s+1, \ldots, t$. (Note that $L^{\alpha} v q \neq 0$ if $r_{\alpha_{v}}>0$.) Then for sufficiently small $\varepsilon>0$ we have $\left|f\left(x_{i}\right)-(p+\varepsilon q)\left(x_{i}\right)\right|=\left|(f-p)\left(x_{i}\right)-\varepsilon q\left(x_{i}\right)\right|<\rho, i=s+1, \ldots, t$. But $q$ and $f-p$ are continuous on $E$ and therefore $\operatorname{sgn} q(x)=\operatorname{sgn}(f-p)(x)$ in a neighborhood $\mathscr{N}$ of $\left\{x_{s+1}, \ldots, x_{t}\right\}$ in $E$. Thus also $|f(x)-(p+\varepsilon q)(x)|<\rho$ in $\mathscr{N}$ for sufficiently small $\varepsilon>0$. Furthermore $|(f-p)(x)|<\rho$ in the compact subset $E-\mathscr{N}$. Thus for sufficiently small $\varepsilon>0,|f(x)-(p+\varepsilon q)(x)|<\rho$ in $(E-\mathscr{N}) \cup \mathscr{N}$ and so $\|f-(p+\varepsilon q)\|<\rho$.

Now we must check that $p+\varepsilon q$ belongs to $V_{0}^{n}$ for $\varepsilon$ small enough and then $p+\varepsilon q$ will furnish a better approximation to $f$ than $p$, yielding the desired contradiction. First, $p+\varepsilon q$ belongs to $V^{n}$. Surely to $\nu=1, \ldots, r$ and sufficiently small $\varepsilon>0$, $l_{\alpha_{v}} \leqq L^{\alpha_{v}}(p+\varepsilon q)=L^{\alpha_{v}} p+\varepsilon L^{\alpha_{v}} q=L^{\alpha_{v}} p-\varepsilon \operatorname{sgn}\left(L^{\alpha_{v}} p-c_{\alpha_{v}}\right) \leqq u_{\alpha_{v}}$; in fact both inequalities are clearly strict if $r_{\alpha_{v}}>0$. But also for any $L^{\alpha}=e_{x_{i}}, i=s+1, \ldots, t$, we have, for sufficiently small $\varepsilon>0$,

$$
\begin{aligned}
l_{\alpha} & <L^{\alpha}(p+\varepsilon q)=L^{\alpha} p+\varepsilon L^{\alpha} q=p\left(x_{i}\right)+\varepsilon q\left(x_{i}\right)=p\left(x_{i}\right)+\varepsilon \operatorname{sgn}\left(f\left(x_{i}\right)-p\left(x_{i}\right)\right) \\
& =L^{\alpha} p-\varepsilon \operatorname{sgn}\left(L^{\alpha} p-c_{\alpha}\right)<u_{\alpha},
\end{aligned}
$$

since $l_{\alpha} \leqq f\left(x_{i}\right) \leqq u_{\alpha}$ and $\left|f\left(x_{i}\right)-p\left(x_{i}\right)\right|=\rho>0$. (Note that $r_{\alpha}>0$.) Let

$$
A^{\prime}=\left(\left\{\alpha_{v}\right\}_{v=1}^{r} \cap\left\{\alpha ; r_{\alpha}>0\right\}\right) \cup\left\{\alpha ; L^{\alpha}=e_{x_{i}}, i=s+1, \ldots, t\right\} .
$$

Then for sufficiently small $\varepsilon>0$, we have $l_{\alpha}<L^{\alpha}(p+\varepsilon q)<u_{\alpha}$ for $\alpha$ in $A^{\prime}$. But by our assumptions, $L^{\alpha} p$ and $L^{\alpha} q$ are continuous on $A$, and $c_{\alpha}$ is continuous on $A$. Hence $\operatorname{sgn} L^{\alpha} q=-\operatorname{sgn}\left(L^{\alpha} p-c_{\alpha}\right)$ in a neighborhood $\mathscr{N}^{\prime}$ of $A^{\prime}$ in $A$. Thus also $l_{\alpha}<L^{\alpha}(p+\varepsilon q)<u_{\alpha}$ in $\mathscr{N}^{\prime}$ for sufficiently small $\varepsilon>0$. Further we have $l_{\alpha}<L^{\alpha} p<u_{\alpha}$ for $\alpha \in A-\mathscr{N}^{\prime \prime}-\mathscr{N}^{\prime}$, where $\mathscr{N}^{\prime \prime}=\left\{\alpha \in A ; r_{\alpha}=0\right\}$. By assumption, $\mathscr{N}^{\prime \prime}$ consists of just isolated points of $A$; thus $\mathscr{N}^{\prime \prime}$ is open in $A$ and so $A-\mathscr{N}^{\prime \prime}-\mathscr{N}^{\prime}$ is compact in $A$. Thus by lower semicontinuity $L^{\alpha} p-l_{\alpha}$ and $u_{\alpha}-L^{\alpha} p$ achieve positive minima on $A-\mathscr{N}^{\prime \prime}-\mathscr{N}^{\prime}$ and so for $\varepsilon$ sufficiently small $l_{\alpha}<L^{\alpha}(p+\varepsilon q)<u_{\alpha}\left(L^{\alpha} q\right.$ is continuous and therefore bounded on $\left.A-\mathscr{N}^{\prime \prime}-\mathscr{N}^{\prime}\right)$. Noting finally that for $\alpha$ in $\mathscr{N}^{\prime \prime}, L^{\alpha} q$ $=-\operatorname{sgn}\left(L^{\alpha} p-c_{\alpha}\right)=0$, we conclude that $l_{\alpha} \leqq L^{\alpha}(p+\varepsilon q) \leqq u_{\alpha}$, for all $\alpha$ in $A$, for sufficiently small $\varepsilon>0$.

THEOREM 3. If $V^{n}$ is generalized Haar with respect to fand $p$, then $p$ is the unique best approximation in $V_{0}^{n}$ to $f$.

Proof. If also $p^{*}$ is a best approximation in $V_{0}^{n}$ to $f$, then so is $p^{* *}=\frac{1}{2} p+\frac{1}{2} p^{*}$, since $V_{0}^{n}$ is convex. Thus there exists an extremal set $\mathscr{S}$ for $f$ and $p^{* *}$ of order $t \geqq n+1$, say $\mathscr{S}=\left\{L^{\alpha_{v}}\right\}_{v=1}^{r} \cup\left\{M^{\beta_{\mu}}\right\}_{\mu=r+1}^{s} \cup\left\{e_{x_{i}}\right\}_{i=s+1}^{t}$. Then $l_{\alpha_{v}} \leqq L^{\alpha_{v}} p^{* *}, L^{\alpha_{v} p}, L^{\alpha_{v}} p^{*}$ 
$\leqq u_{\alpha_{v}}$, and $L^{\alpha_{v}} p^{* *}=l_{\alpha_{v}}\left(u_{\alpha_{v}}\right)$ therefore implies that $L^{\alpha_{v} p}=L^{\alpha_{v}} p^{*}=l_{\alpha_{v}}\left(u_{\alpha_{v}}\right), \nu=1, \ldots, r$. As a result, $M^{\beta_{\mu}} p=M^{\beta_{\mu}} p^{*}=m_{\beta_{\mu}}\left(n_{\beta_{\mu}}\right), \mu=r+1, \ldots, s$. Finally $\left|f\left(x_{i}\right)-p^{* *}\left(x_{i}\right)\right|$ $=\left\|f-p^{* *}\right\|=\|f-p\|=\left\|f-p^{*}\right\|$ implies $p\left(x_{i}\right)=p^{*}\left(x_{i}\right), i=s+1, \ldots, t$. But since $t \geqq n+1$ and some $n$ of the elements of $\mathscr{S}$ are linearly independent in the dual of $V^{n}$, we have $p=p^{*}$.

COROLlaRY 1. If $V^{n}$ is generalized Haar, then any best approximation $p$ in $V_{0}^{n}$ to $f$ is unique.

3. Restricted derivatives approximation. We now show that in Example 3 the generalized Haar condition holds on $V^{n}=P_{n-1}$ and therefore the results of $\S 2$ are valid in this case.

Fix $\left\{x_{1}, x_{2}, \ldots, x_{k}\right\} \subset[a, b]$. Let $\mathscr{L}_{i}^{j}$ denote the linear functional on $P_{n-1}$ defined by $\mathscr{L}_{i}^{j} p=p^{(j)}\left(x_{i}\right)$. Following Schoenberg [12], let $\mathscr{E}=\left(e_{i j}\right)_{i=1,2, \ldots ;, k}^{j-1}$ be an $n$ incidence matrix, i.e. each $e_{i j}$ is 0 or 1 and $\sum_{(i, j)} e_{i j}=n$. $\mathscr{E}$ is said to be poised if the set of $n$ linear functionals $\left\{\mathscr{L}_{i}^{j} ; e_{i j}=1\right\}$ is linearly independent on $P_{n-1}$. If $\mathscr{E}$ is an $n$-incidence matrix, let $m_{j}=\sum_{i=1}^{k} e_{i j}, j=0,1, \ldots, n-1$, and $M_{j}=\sum_{p=0}^{j} m_{p}, j=0,1$, $\ldots, n-1$. Then $\mathscr{E}$ is said to satisfy the Pólya conditions if $M_{j} \geqq j+1$ for $j=0,1, \ldots$, $n-1$.

In the following four statements of theorems, suppose that the $n$-incidence matrix $\mathscr{E}$ satisfies the Pólya conditions.

TheOREM A (Pólya AND WhitTAKer, SEe [3]). If $k=2$, then $\mathscr{E}$ is poised.

TheOREM B (Ferguson [3, P. 24]). If $k>2$ and if $e_{i, j-1}=e_{i, j+p}=0, e_{i j}=\cdots$ $=e_{i, j+p-1}=1$ implies $p$ is even, then $\mathscr{E}$ is poised.

TheOREM C (SCHOENBERG, SEE [3, P. 25]). If $x_{1}=a$ and $x_{k}=b$, and if $2 \leqq i \leqq k-1$ and $e_{i j}=1$ imply $e_{i j^{\prime}}=1$ for each $j^{\prime} \leqq j$, then $\mathscr{E}$ is poised.

By combining the proofs of Theorems $\mathrm{B}$ and $\mathrm{C}$ given by Ferguson, we can get the following result, which has also been given by Atkinson and Sharma [1].

THEOREM D. If $x_{1}=a$ and $x_{k}=b$ and if $2 \leqq i \leqq k-1$ and $e_{i, j-1}=e_{i, j+p}=0$, $e_{i j}=\cdots=e_{i, j+p-1}=1$ imply $p$ is even, then $\mathscr{E}$ is poised.

THEOREM 4. $P_{n-1}$ satisfies the generalized Haar condition in the case of restricted derivatives approximation.

Proof. Let $\mathscr{S}$ be the maximal extremal set for $f$ and $p$. Now create an incidence matrix $\mathscr{E}=\left(e_{i j}\right)_{i=1,2, \ldots, k}^{j}, \ldots, k$ representing the occurrence of elements of $\mathscr{S}$ as follows. If $m_{j}=\sum_{i=1}^{k} e_{i j}, j=0,1, \ldots, n-1$, then $m_{0}$ is as large as possible but smaller than $n+1$ and, inductively, given $m_{0}, m_{1}, \ldots, m_{j-1}$, we take $m_{j}$ as large as possible, but smaller than $n+1, j=1,2, \ldots, n-1$. We also insist that $\mathscr{E}$ satisfy the conditions of Theorem $\mathrm{D}$ except of course that $\mathscr{E}$ need not have exactly $n$ 1-entries in its first $n$ columns and except possibly for the Pólya conditions $\left(\sum_{p=0}^{j} m_{p} \geqq j+1, j=0,1\right.$, 
$\ldots, n-1)$. This can always be accomplished by adding, if necessary, 1's to $\mathscr{E}$ representing $M^{(x, i)}$ 's in $\mathscr{S}$.

We claim that $\mathscr{E}$ in fact satisfies the strong Pólya conditions $\left(\sum_{p=0}^{j} m_{p} \geqq j+2\right.$, $j=0,1, \ldots, n-2)$. Suppose on the contrary that $\sum_{p=0}^{j} m_{p} \geqq j+2$ for $j=0,1, \ldots, r-1$, $r<n-1$, but $\sum_{p=0}^{r} m_{p}<r+2$ where $k_{s-1} \leqq r<k_{s}$ (take $\left.k_{q+1}=\infty\right)$. Now let $p(x)$ $=p_{1}(x)+p_{2}(x)$, where $p_{1}(x)=a_{0}+a_{1} x+\cdots+a_{r} x^{r}$ and $p_{2}(x)=a_{r+1} x^{r+1}+\cdots$ $+a_{n-1} x^{n-1}$. Then $p_{1}(x)$ is clearly a best approximation to $f(x)-p_{2}(x)$ in the set $V_{0}^{r+1}=\left\{p \in P_{r} ; l_{i}(x)-p_{2}^{\left(k_{i}\right)}(x) \leqq p^{\left(k_{i}\right)}(x) \leqq u_{i}(x)-p_{2}^{\left(k_{i}\right)}(x), i=0,1, \ldots, s-1\right\}$ with a maximal extremal set $\mathscr{S}_{1}$ consisting of the $t=\sum_{p=0}^{r} m_{p}$ elements of $\mathscr{S}$ represented by the 1 -entries in the first $r+1$ columns of $\mathscr{E}$. But $t \leqq r+1$ and so by Theorem $\mathrm{D}$ these $t$ elements in $\mathscr{S}_{1}$ are linearly independent. Thus $V^{r+1}=P_{r}$ is generalized Haar with respect to $f-p_{2}$ and $p_{1}$ and so Theorem 2 applies to show that in fact $t \geqq r+2$. Thus $\mathscr{E}$ satisfies the strong Pólya conditions.

Now it is easy to see that $\mathscr{E}$ can be "trimmed down" to an $n$-incidence matrix satisfying the Pólya conditions. This is accomplished by changing pairs of consecutive 1's to 0 's in the rows, beginning over in the $(n+1)$ th column (recall $\mathscr{E}$ originally has $n+1$ columns) and working backwards column by column until there are $n$ or $n+11$ 's left. At this stage $\mathscr{E}$ still satisfies the strong Pólya conditions, as is easily seen. If there are $n+11$ 's left at the completion of the process, then further delete a 1 from the end of a consecutive string of 1 's in a row beginning in the 0 th column. This last step may of course result in $\mathscr{E}$ not satisfying the strong Pólya conditions but clearly $\mathscr{E}$ will still satisfy the Pólya conditions.

In this way we obtain an $n$-incidence matrix $\mathscr{E}$ of elements in $\mathscr{S}$ which satisfies the Pólya conditions and the conditions of Theorem D and thus is poised. We conclude that $\mathscr{S}$ contains $n$ elements which are linearly independent in the dual of $P_{n-1}$ and the result follows.

COROLLARY 2. In the case of restricted derivatives approximation, if the approximating subset is not empty, then there always exists a unique best approximation $\left.{ }^{1}\right)$.

4. Remark. The results of this paper are also valid if, instead of the supremum norm, we measure the error by Moursund's generalized weight function [8], or Dunham's ordering function [2], or Griesel's error function [4].

\section{REFERENCES}

1. K. Atkinson and A. Sharma, A partial characterization of poised Hermite-Birkhoff interpolation problems, SIAM J. Numer. Anal. 6 (1969), 230-235. MR 41 \#9419.

2. C. B. Dunham, Chebyshev approximation with respect to a weight function, J. Approximation Theory 2 (1969), 223-232. MR 40 \#6137.

3. David Ferguson, The question of uniqueness for G. D. Birkhoff interpolation problems, J. Approximation Theory 2 (1969), 1-28. MR 40 \#599.

(1) Professors J. A. Roulier and G. D. Taylor have independently obtained this result in a paper entitled Approximation by polynomials with restricted ranges on their derivatives, currently submitted for publication. 
4. M. A. Griesel, Uniform approximation with respect to a general error function, Doctoral Dissertation, University of California, Riverside, Calif., 1971.

5. H. L. Loeb, D. G. Moursund, L. L. Schumaker and G. D. Taylor, Uniform generalized weight function polynomial approximation with interpolation, SIAM J. Numer. Anal. 6 (1969), 284-293. MR 40 \#3679.

6. G. G. Lorentz and K. L. Zeller, Monotone approximation by algebraic polynomials, Trans. Amer. Math. Soc. 149 (1970), 1-18.

7. R. A. Lorentz, Uniqueness of approximation by monotone polynomials (to appear).

8. D. G. Moursund, Chebyshev approximation using a generalized weight function, SIAM J. Numer. Anal. 3 (1966), 435-450. MR 34 \#4771.

9. T. J. Rivlin and H. S. Shapiro, $A$ unified approach to certain problems of approximation and minimization, SIAM J. Numer. Anal. 9 (1961), 670-699. MR 24 \#A3462.

10. J. A. Roulier, Monotone approximation of certain classes of continuous functions, $\mathrm{J}$. Approximation Theory 1 (1968), 319-324. MR 38 \#4875.

11. J. A. Roulier and G. D. Taylor, Uniform approximation by polynomials having bounded coefficients, Abh. Math. Sem. Univ. Hamburg 36 (1971), 126-135.

12. I. J. Schoenberg, On Hermite-Birkhoff interpolation, J. Math. Anal. Appl. 16 (1966), 538-543. MR 34 \#3160.

13. O. Shisha, Monotone approximation, Pacific J. Math. 15 (1965), 667-671. MR 32 \#2802.

14. G. D. Taylor, On approximation by polynomials having restricted ranges, SIAM J. Numer. Anal. 5 (1968), 258-268. MR 40 \#6142.

15. - Approximation by functions having restricted ranges. III, J. Math. Anal. Appl. 27 (1969), 241-248. MR 41 \#2261.

Department of Mathematics, University of California, Riverside, California 92502 